\title{
Long-term sick-leavers with fibromyalgia: Comparing their multidisciplinarily assessed characteristics with those of others with chronic pain conditions and depression
}

\author{
Jürgen Linder ${ }^{\prime}$ \\ Kristina Schüldt Ekholm²,3,4 \\ Göran Lundh' \\ Jan Ekholm ${ }^{2,3}$ \\ 'Diagnostic Centre, Division \\ of Psychiatry, Department of Clinical \\ Neuroscience, Karolinska Institutet, \\ Stockholm, Sweden; ${ }^{2}$ Division \\ of Rehabilitation Medicine, \\ Department of Clinical Sciences, \\ Danderyd Hospital, Karolinska \\ Institutet, Stockholm, Sweden; \\ ${ }^{3}$ Stockholm University Rehabilitation \\ Medicine Clinic, Danderyd Hospital, \\ Stockholm, Sweden; ${ }^{4}$ Division of \\ Rehabilitation Science, Department \\ of Health Science, Mid-Sweden \\ University, Campus Östersund, \\ Sweden
}

Objective: The aim was to gain knowledge of fibromyalgia (FM) patients on long-term sick leave and with particular difficulties in resuming work, and to compare them with patients with myalgia, back or joint diagnoses, and depression.

Methods: Patients were identified by and referred from social insurance offices and were multidisciplinarily examined by three board-certified specialists in psychiatry, orthopedic surgery and rehabilitation medicine. Ninety-two women were diagnosed with FM only. Three female comparison groups were chosen: depression, back/joint diagnoses, and myalgia.

Results and conclusions: Ceaseless pain was reported by $73 \%$ of FM patients, $54 \%$ of back/joint diagnoses patients, $43 \%$ of myalgia patients, and $35 \%$ of depression patients. The distribution of pain $(>50 \%)$ in FM patients was to almost all regions of the body, and in depression patients to the lower dorsal neck, upper shoulders and lumbosacral back but not in the anterior body. Reduced sleep was more evident in FM patients. FM patients did not meet more criteria for personality disorder than patients with the other somatic pain conditions. The most common dimension of "personality traits" of somatic pain conditions was the "obsessive compulsive" but at a level clearly below that indicating a personality disorder. More FM patients experienced disabilities, the most common being in the mobility and domestic-life areas.

Keywords: fibromyalgia, long-term sick-leave, multidisciplinary assessment, depression, chronic pain conditions, disability, pain distribution

\section{Introduction}

In a multidisciplinary study of long-term sick-leavers with particular difficulty in resuming work, fibromyalgia (FM) was the most common somatic diagnosis. ${ }^{1}$ Patients with FM constitute many of those outside working life in Western countries including Sweden, as a result of long-term sickness absence or the receipt of a disability pension. ${ }^{2-4}$

Fibromyalgia is currently a well-known prevalent, chronic-pain condition in which central nervous system (CNS) sensitization is believed to be one of the important mechanisms causing increased generalized pain sensitivity which may lead to maintenance of the pain. ${ }^{5-8}$ Changes have also been shown in other systems, including muscles ${ }^{9,10}$ and the neuro-endocrinological system. ${ }^{11-14}$ Depression is also common in patients with chronic pain conditions. ${ }^{15,16}$

Recent studies have shown positive effects on FM with the antiepileptic pregabalin, ${ }^{17}$ and the serotonin and norepinephrine reuptake inhibitors (SNRIs) duloxetine and venlafaxine, ${ }^{18}$ which has stimulated new clinical interest in the syndrome. Clinicians need to recognize the characteristics of this kind of patient, with FM and on long-term sick leave, who will often need special treatment and rehabilitation to end their sick leave. 
Although the FM syndrome has been extensively described, ${ }^{19-22}$ the present patients were all on long-term sick leave with marked difficulty in resuming work.

The rationale for this study was to analyze in further detail the characteristics of long-term sick-listed patients with FM, to establish further guidelines for clinicians in the management of these patients.

\section{Aim}

The aim was to use data from multidisciplinary assessment for long-term sick-leavers with particular difficulties in resuming work in order to gain more knowledge of FM patients. Comparison groups were patients with myalgia, back or joint diagnoses, and depression who were also long-term sickleavers with particular difficulties in resuming work, and who had also been assessed in a multidisciplinary clinical setting.

These specific questions were addressed:

1. What are the typical symptoms/impairments in long-term sick-listed FM patients?

2. What are the typical somatic and psychiatric symptoms in FM patients in comparison with other groups studied?

3. What are the common denominators and differences in and between FM patients and patients with depression?

4. Are patients with FM associated with any personality disorder or specific personality traits compared with other groups studied?

5. What is the typical pattern of activity limitations in FM?

6. What physical activity did FM patients do before age 30 in comparison with other groups studied?

7. What are their present levels of physical activity in comparison with other groups studied?

8. Are there changes in leisure activities in FM patients in comparison with other groups studied?

9. What are the characteristics of pain descriptors; present pain, intensity, pain-free periods in comparison with other groups studied?

10. What is the pattern of pain distribution in FM patients compared with patients diagnosed with myalgia, back/ joint diagnoses, or depression?

11. What is the state of FM patients' health-related quality of life?

12. What proportion of the FM patients had a local pain condition before FM developed?

\section{Material and methods}

\section{Subjects}

Long-term sick-leavers with extreme difficulty in resuming working life were identified by local social insurance offices in Sweden's Stockholm County, which has a population of about two million. They were referred by insurance office officials to a special unit, the Diagnostic Centre at the Karolinska Hospital, Stockholm, for diagnosis of somatic and psychiatric diseases and disorders, and evaluation of work ability. All sick-leavers were accepted, resulting in 640 patients, 416 women and 224 men, being consecutively investigated in a multidisciplinary clinical setting. The clinical aims were to establish diagnoses and prognoses, to assess the patients' current disability status, and to recommend possible rehabilitation, ie, medical, vocational, or combined.

A particular aim of the current research was to further examine a subsample of patients diagnosed multidisciplinarily with FM. Of the 640 patients, 93 were diagnosed with FM (92 women and one man). Because only one male patient was diagnosed with FM, we chose all 416 women and no males. From this subgroup, three comparison groups were chosen with respect to the diagnoses established after the clinical assessments in our unit: a) female patients with depression but no somatic diagnoses, $\mathrm{n}=169$; $\mathrm{b}$ ) female patients with diagnoses in back or joints (ICD-10 code M00-M25; M40-M54 except the more unspecific diagnoses M545 and M549, lumbago and unspecified back pain), $\mathrm{n}=111$, and c) patients diagnosed as having myalgia (M60-M79, except FM), $n=44$. All patients studied were females, because only one male of the 640 patients studied met the diagnosis criteria for FM. The corresponding diagnoses in the male subgroup which is not presented in this paper, were depression, $n=140$, diagnoses in back or joints, $n=66$, myalgia, $n=15$, and FM $n=1$.

About half the patients had white collar types of work and there were no statistical differences between the groups.

\section{Clinical setting}

All the patients were examined by three board-certified specialists in psychiatry, orthopedic surgery and rehabilitation medicine, respectively, at a diagnostic centre at the Karolinska Hospital, Stockholm, Sweden. This unit specializes in multidisciplinary investigation of patients on long-term sick leave.

\section{Procedures, measures and assessments}

The procedures below were used for all patients as part of the multidisciplinary disability evaluation.

At their first visit, the patients completed five questionnaires and self-report scales.

\section{Patient history}

Data on education, social status, occupation, work were gathered. Questions elicited information on pain (onset, duration, frequency, 
intensity), defined current symptoms (yes/no), and defined current functional impairments (yes/no). Further questions covered problems with sleep, leisure activities, and physical activity level before age 30 and at present. In addition, during the interview, all patients were asked about the debut of their current pain condition.

\section{Psychiatric self-report questionnaires Symptoms}

The Comprehensive Psychopathological Rating Scale for Self Administration (CPRS-S-A), measuring 23 symptoms and including the Montgomery-Åsberg Depression Rating Scale (MADRS). ${ }^{23}$

\section{Personality disorders}

The Structured Clinical Interview for Diagnosis (SCIDII-Screen) Questionnaire covering criteria for avoidant, dependent, obsessive-compulsive, paranoid, schizoid, schizotypal, borderline, histrionic, and narcissistic personality disorders in DSM-IV. ${ }^{24}$

\section{The Depression Rating Self-Report Questionnaire (DSRS) ${ }^{25}$}

\section{Quality of life}

The Rand 36-item Health Survey (SF36)

The SF36 is a measure of functional impairment and wellbeing. It assesses eight domains: physical functioning, role limitations due to physical health problems, bodily pain, vitality, general health perception, social functioning, role limitations due to emotional problems, and perceived mental health. ${ }^{26,27}$

\section{Pain assessments}

Pain intensity

Pain intensity was estimated with visual analogue scales (VAS) for worst and least pain during the previous two months, and present level of pain. Pain was further assessed with the pain item "aches and pains" in the CPRS-SA ${ }^{23}$ and using pain questions in the medical history questionnaire (presence, frequency, duration, localization) and the pain item in SF-36.

\section{Pain drawings}

The subjects indicated the location of their pain on diagrams comprising 57 defined body regions. Twenty-three areas covered the neck-shoulder-upper-extremity region, nine the lumbosacral spine-thigh region and 12 the knee-lower-leg-foot region. Categorization was based on the number of subjects who had made a pain mark in each of the 57 areas. $^{28}$

\section{Sick-leave, disability pension}

Employment/insurance-benefit status was reported by the client and also by the social insurance office. In addition, data on the patient's periods of sick-leave and/or partial (temporary or permanent) disability pension were provided by the referring insurance office. There were few discrepancies. When in doubt, we used data from the social insurance office. The proportions in the material of the various forms of benefit are shown in Table 1. When referred to the multidisciplinary unit, two-thirds of the patients were on a sickness allowance and about one-fourth on a temporary disability pension.

To interpret figures on health-insurance status, we should note that in Sweden, partial benefits are available for sickness allowances, and temporary or permanent disability pensions correspond to $1 / 4,1 / 2$, and $3 / 4$ of working incapacity, with reference to prior ordinary work hours. This means that a patient can have a half-time, temporary disability pension and simultaneously receive a sickness allowance for the remaining half, if unable to work as a result of a relapse of the original condition, a complication or another disease. These arrangements explain why some of our patients were sick-listed with a sickness allowance and concomitantly received a temporary disability pension or in a few cases even a permanent disability pension. The patients had been receiving benefits for one year or more preceding the current investigation.

Table I Patient subgroups based on diagnoses: fibromyalgia, myalgia, diseases in spine/joints, and depression. Number of patients in each group, mean age (years, SD); percent higher level of education and percent fully employed

\begin{tabular}{lllll}
\hline Patient group diagnoses & Number of patients $\mathbf{n}$ & Mean age years (SD) & Higher education \% & Employed \% \\
\hline Fibromyalgia & 92 & $45.6(10.2)$ & 66.3 & 40.2 \\
Myalgia & 44 & $44.4(8.1)$ & 63.6 & 52.3 \\
Spine/Joints & 111 & $46.4(8.2)$ & 68.5 & 56.8 \\
Depression & 169 & $46.5(9.5)$ & 73.4 & 54.4 \\
\hline
\end{tabular}

Notes: There was no significant difference in mean age or educational level between the groups. Fever patients in the fibromyalgia group were employed, but the difference was not significant $(p=0.0885$, chi-square test). 


\section{Clinical procedure}

Before their first visit the patients were informed by letter about the aim and procedures of the investigation. On a second occasion they were contacted by phone and could ask about the purpose and conduct of the investigation. This procedure was adopted because many patients felt disturbed or insulted by the referral from the social insurance authorities. Further, at the patients' first visit before the investigation started they were informed once more about the aim and conduct of the investigation by a Diagnostic Centre staff member. They were also encouraged to ask further questions while filling in the questionnaires at the Centre.

Filling in questionnaires "in situ", with the opportunity of asking questions of the staff, meant that few questionnaires were incomplete.

Data from all questionnaires were immediately transferred into a 4D-database ${ }^{29}$ before the clinical assessment. Individual protocols from the questionnaires were printed out and given to the specialists for their guidance, and to verify the accuracy of each patient's input data. Moreover, the database was especially constructed to enable output of summarized data research purposes.

Each patient was investigated during three separate visits within three weeks. During the first visit and after completing the questionnaires, the patients underwent a full psychiatric evaluation by a board-certified psychiatrist, who had access to the results of the questionnaires. During this evaluation, patients were given feedback on the questionnaire results. The psychiatric results, the diagnoses obtained and the psychiatrist's assessment of current disability, and recommendation for possible psychiatric rehabilitation, were documented in the patient's record.

At each patient's second visit an orthopedic evaluation was performed by a board-certified orthopedic surgeon, who had access to the previous psychiatric record and to previous orthopedic opinions and X-rays. The orthopedic results (diagnoses, current disability, recommendations for rehabilitation) were documented as described above.

The patient's third visit comprised a full rehabilitation and medical evaluation by a certified rehabilitation-medicine physician who had access to the preceding documentation. The results of this third evaluation were also documented as above.

The diagnosis of FM was based on the American College of Rheumatology (ACR) criteria. ${ }^{20}$ The diagnosis of myalgia was given when the muscular pain was local and the painful muscle or muscle group was marked off from its surroundings, and the basic criteria for direct and indirect pain were met.

Finally, the results of the three evaluations were discussed for each patient with all three specialists present, resulting in a joint statement on the diagnosis, and degree and duration of disability, mainly relating to working capacity. Recommendations for possible rehabilitation measures were also made. The statement was returned to the referring local Social Insurance Office.

\section{Statistical methods}

Means, medians and standard errors of mean were used for descriptive statistics. Non-parametric statistics (the chi-square test and Wilcoxon's rank sum test) were used for comparisons between and within groups. Statistics were calculated using the JMP statistical software. ${ }^{30}$ Unless otherwise stated, $\mathrm{p} \leq 0.05$ was chosen as the level of statistically significant difference. To control for falsepositive results due to mass significance, the Tukey-Kramer correction was used.

\section{Ethical approval}

The study was approved by the Stockholm Regional Ethics Committee (DNR 95-149; 2006/1281-31; 2008/2-7).

\section{Results}

\section{What are the typical symptoms/ impairments in long-term sick-listed FM patients?}

Figure 1 shows that more than 95\% of patients with FM reported symptoms of tiredness, headaches, muscular tension, muscular weakness, muscular tiredness, and muscular stiffness on awakening.

\section{What are the typical somatic and psychiatric symptoms in FM patients?}

A statistical analysis of the symptoms showed no statistical differences for any of the symptoms between the groups with spinal/joint disorders and patients with myalgia. Thus, these two groups were pooled. The symptoms were compared between three groups: patients with depression (D), $n=169$; patients with $\mathrm{FM}, \mathrm{n}=92$, and patients with myalgia or spinal/ joint disorders ( $\mathrm{S}$ ), $\mathrm{n}=155$ (Figure 2).

Significant differences $(\mathrm{P}<0.0001$, chi-square test $)$ between all three groups were noted for: reduced sleep $(\mathrm{FM}>\mathrm{D}>\mathrm{S}$ ), inner tension $(D>F M>S$ ), worrying over trifles $(D>F M>S$ ), lassitude $(\mathrm{D}>\mathrm{FM}>\mathrm{S})$, and indecision $(\mathrm{D}>\mathrm{FM}>\mathrm{S})$. 


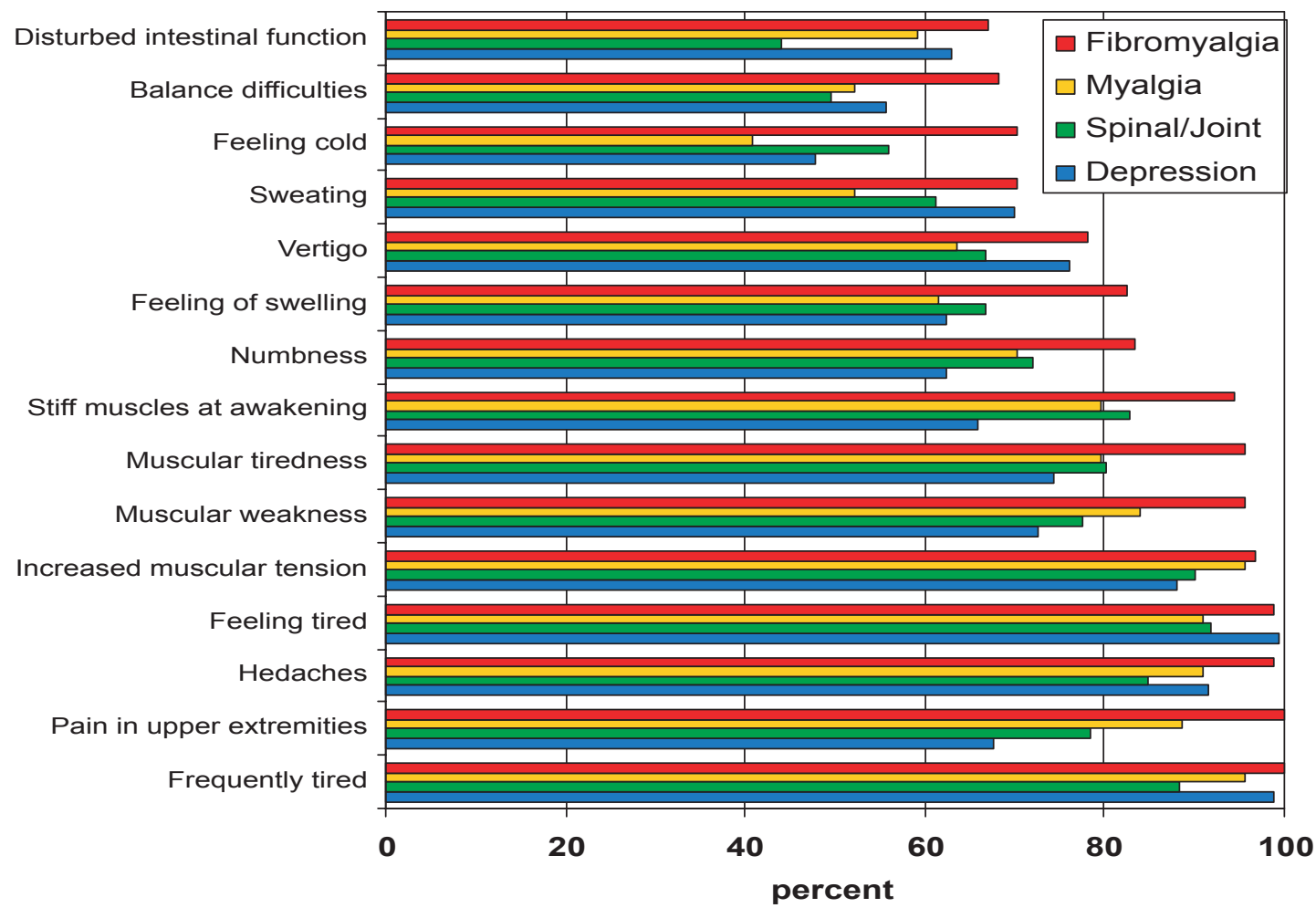

Figure I Frequency of reported specific symptoms/functional impairments in patients with fibromyalgia (FM), myalgia, back/joint diagnoses, and depression sorted by increasing frequency of FM patients.

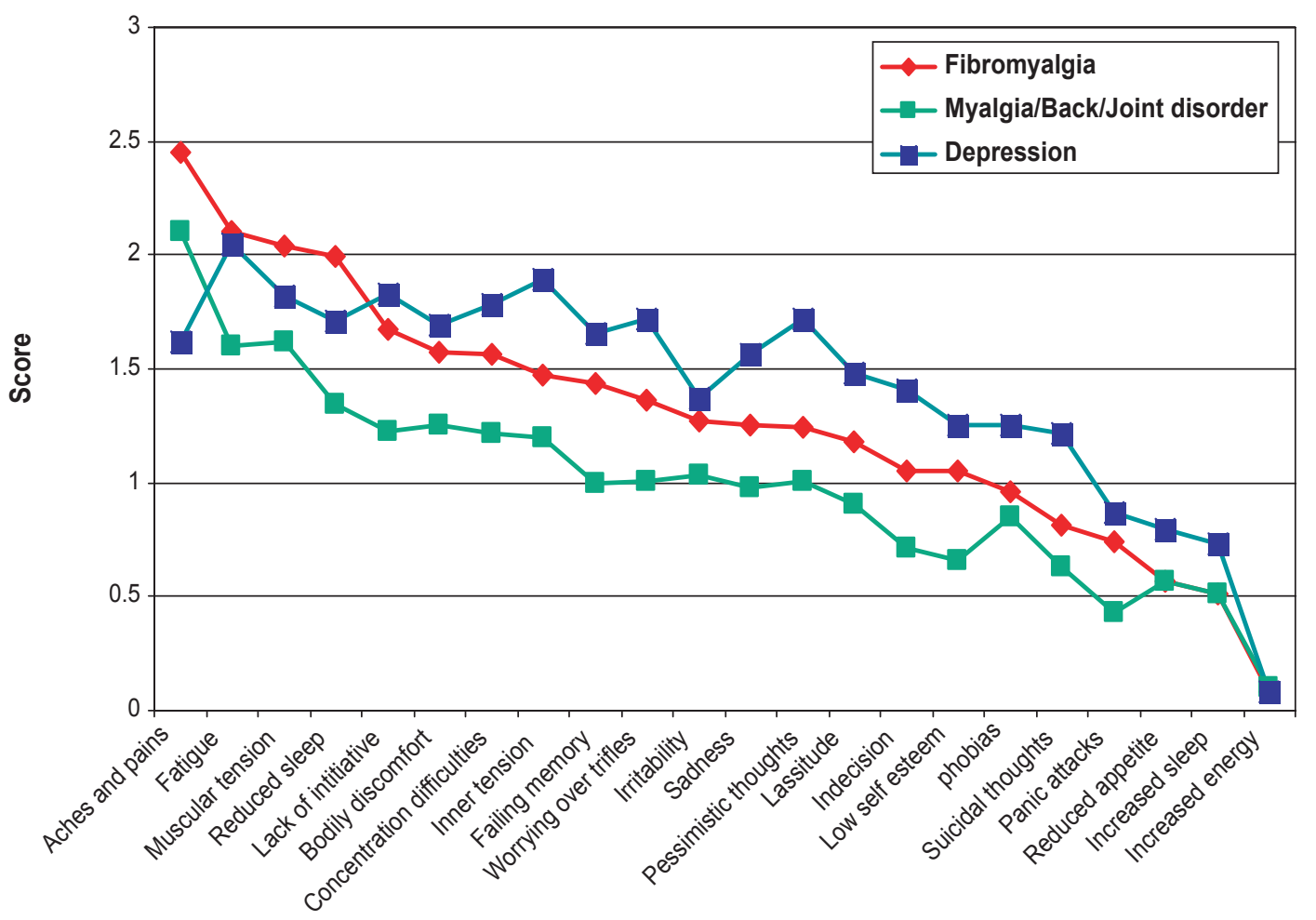

Figure 2 Symptom profile in patients with fibromyalgia (FM), myalgia, back/joint diagnoses, and depression. Symptoms along horizontal axis and scores, $0=$ none, I = mild, 2 = moderate, 3 = severe, on vertical axis. Symptoms are sorted by decreasing FM scores. 


\section{Symptoms in FM compared with depression and somatic disorders}

Higher scores ( $\mathrm{p}<0.0001)$ for FM compared with depression were noted for two items: aches and pains, reduced sleep.

Higher scores $(p<0.0001)$ for FM compared with the group with other somatic disorders were noted for: muscular tension, lassitude, bodily discomfort, concentration difficulties, inner tension, failing memory, worrying over trifles, pessimistic thoughts, indecision, low self esteem, and panic attacks.

\section{Symptoms in depression compared with FM and somatic disorders}

Higher scores $(\mathrm{p}<0.0001)$ for depression compared with FM were noted for: inner tension, worrying over trifles, sadness, pessimistic thoughts, lassitude, indecision, suicidal thoughts, and reduced appetite.

Similar item scores for depression and FM were noted for: fatigue, muscular tension, bodily discomfort, concentration difficulties, failing memory, irritability, low self-esteem, phobias, panic attacks, increased sleep, and increased energy.

In summary, the FM patients had higher scores for aches and pains, reduced sleep, and perceived muscular tension, while the depression group had higher scores for the 15 other symptoms: lack of initiative, bodily discomfort, concentration difficulties, inner tension, failing memory, irritability, sadness, pessimistic thoughts, lassitude, indecision, low self-esteem, phobias, suicidal thoughts, panic attacks, and increased sleep (Figure 2).

For these 15 symptoms the FM patients scored in between the depression patients and the myalgia/back/joint patients.

\section{What are the common denominators and differences in and between FM patients and patients with depression?}

Among patients with depression, 88\% met the self-reported diagnostic criteria for major depression, compared with $59 \%$ in FM, 55\% in myalgia, and $46 \%$ in those with back/joint diagnoses. A clinical diagnosis of depression was recorded in $100 \%$ of the depression group, $52 \%$ of the FM patients, $36 \%$ of the myalgia patients, and $34 \%$ of back/joint patients. Almost all patients with FM reported reduced energy, reduced sleep, and concentration difficulties, ie, three of nine criteria for the diagnosis of major depression according to the Diagnostic and Statistical Manual of Mental Disorders 4th edition (DSM-IV). For this diagnosis, five criteria are needed (Figure 3).

\section{Are patients with FM associated with any personality disorder or specific personality traits?}

As expected, more criteria for all DSM-IV personalitydisorder dimensions were noted in patients with depression

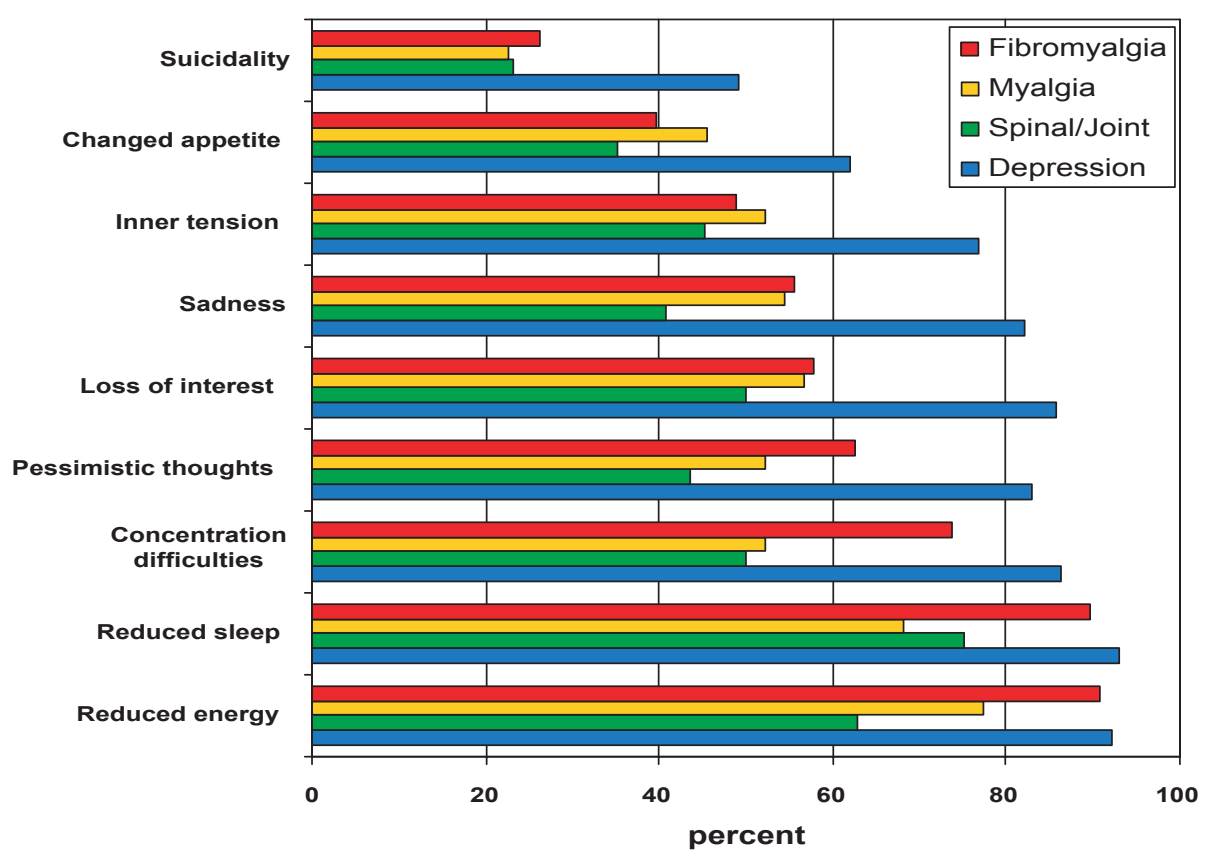

Figure 3 Distribution of criteria for DSM-IV major depressive episode in patients clinically diagnosed as fibromyalgia (FM), myalgia, back/joint diagnoses, and depression, sorted by increasing frequency of criteria in FM patients. 
compared with the three patient groups with somatic diagnoses. The latter as a group had the highest average number of criteria for obsessive compulsive (anancastic) personality traits seen as a dimension, though clearly below the cutoff for any personality disorder (Figure 4).

\section{What is the typical pattern of limitations in activities in FM?}

The most frequent disabilities in patients with FM, reported by more than two of three patients, involved carrying bags with foodstuffs, vacuum cleaning, hanging up laundry, turning the head, peeling potatoes, picking up objects from floor, washing up by hand, holding objects, and hair washing (Figure 5).

\section{What physical activities did FM patients do before the age of 30 ?}

The present patient groups were not characterized by low physical activity before the age of 30 years and there was no difference between the groups studied. Indeed $70 \%-85 \%$ of all patients reported that they had been active in training or participated as "elite" or at least at district level in competition below the age of 30 years (Figure 6).

\section{What are their present levels of physical activity?}

About $20 \%$ of the patients with myalgia or back/joint disorders reported that they were physically active for more than three hours daily. About $15 \%$ of the patients with depression, and $10 \%$ of the FM patients reported this level of present physical activity (Figure 7).

\section{Are there changes in leisure activities in FM patients?}

All patients had changed their activity level and had fewer activities at present. The number of patients who had ceased all activities was higher (30\%) for patients with FM or with depression than for those with other somatic disorders, where about $20 \%$ had ceased all activities (Figure 8).

\section{What are the characteristics of pain descriptors; present pain, intensity, pain-free periods? \\ Present pain}

Ongoing pain was reported by $100 \%$ of the patients with FM and myalgia, $98 \%$ of patients with back/joint diagnoses, and

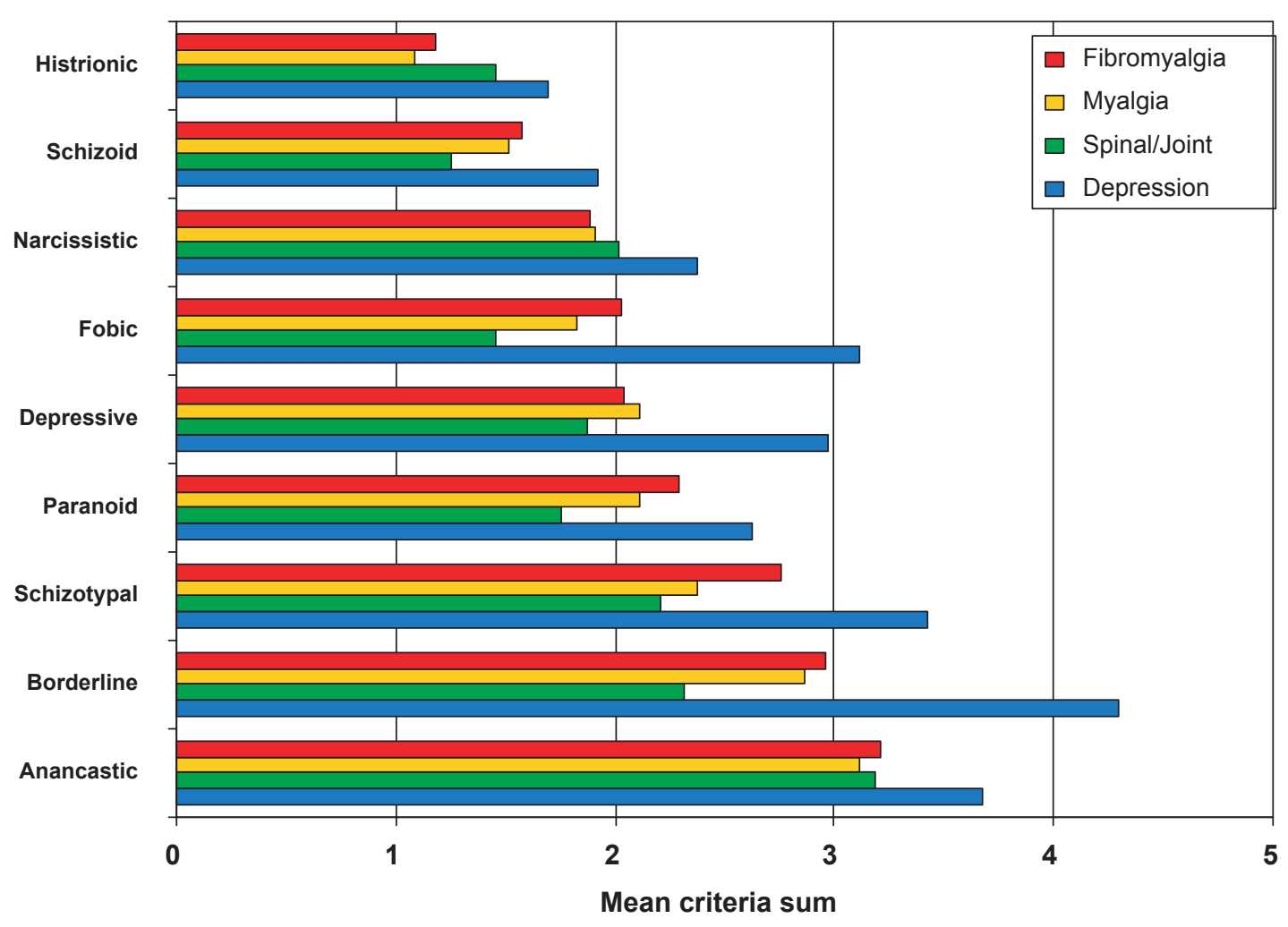

Figure 4 Means of criteria sums for DSM-IV personality disorders in patients with fibromyalgia (FM), myalgia, back/joint diagnoses, and depression ordered by increasing frequency of criteria in FM patients. 


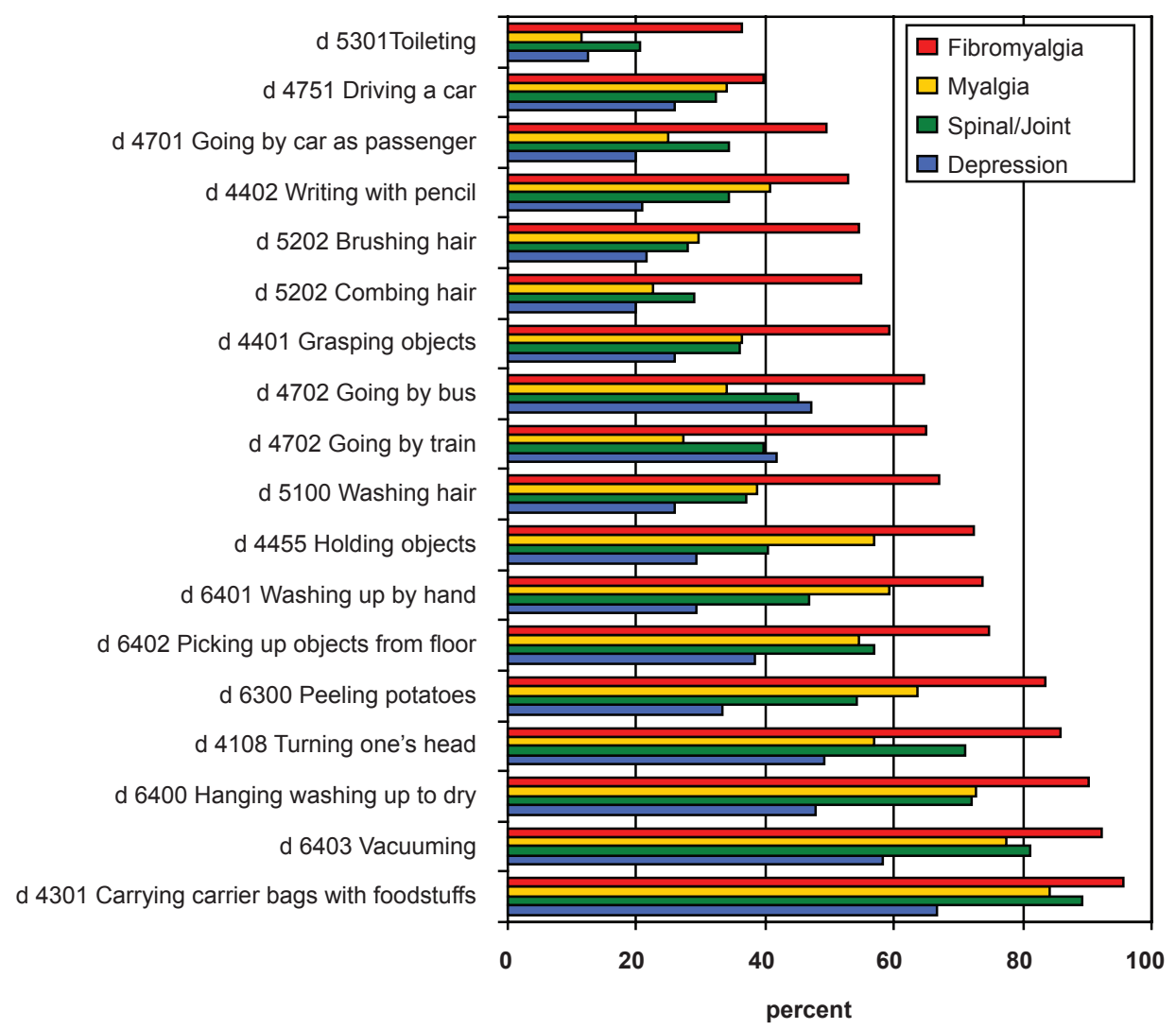

Figure 5 Frequency of reported difficulties in activities (ICF numbers ${ }^{31}$ and description) in patients with fibromyalgia, myalgia, back/joint diagnoses, and depression sorted in increasing frequency for fibromyalgia patients.

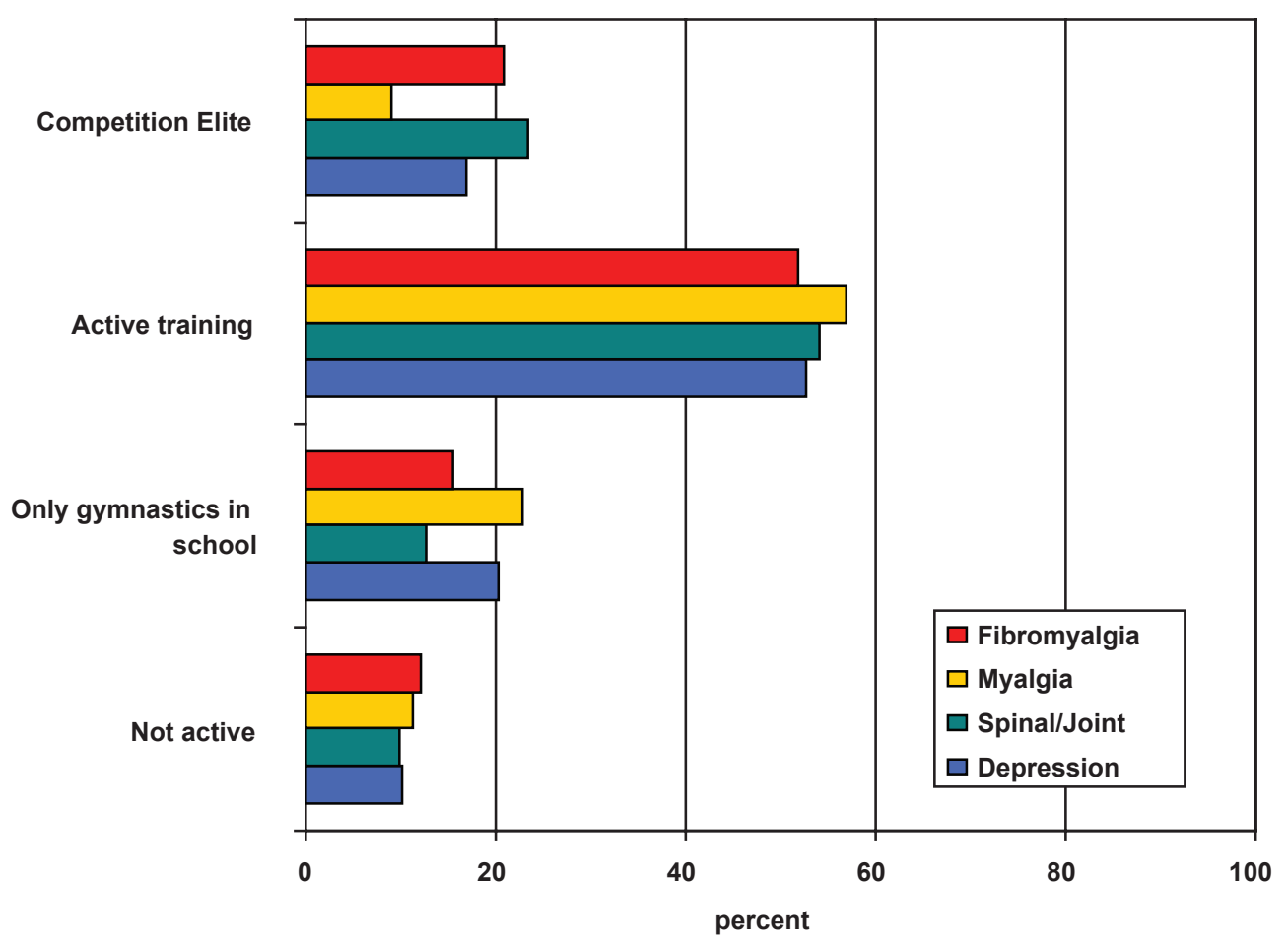

Figure 6 Patterns of physical activity before age 30 years. 


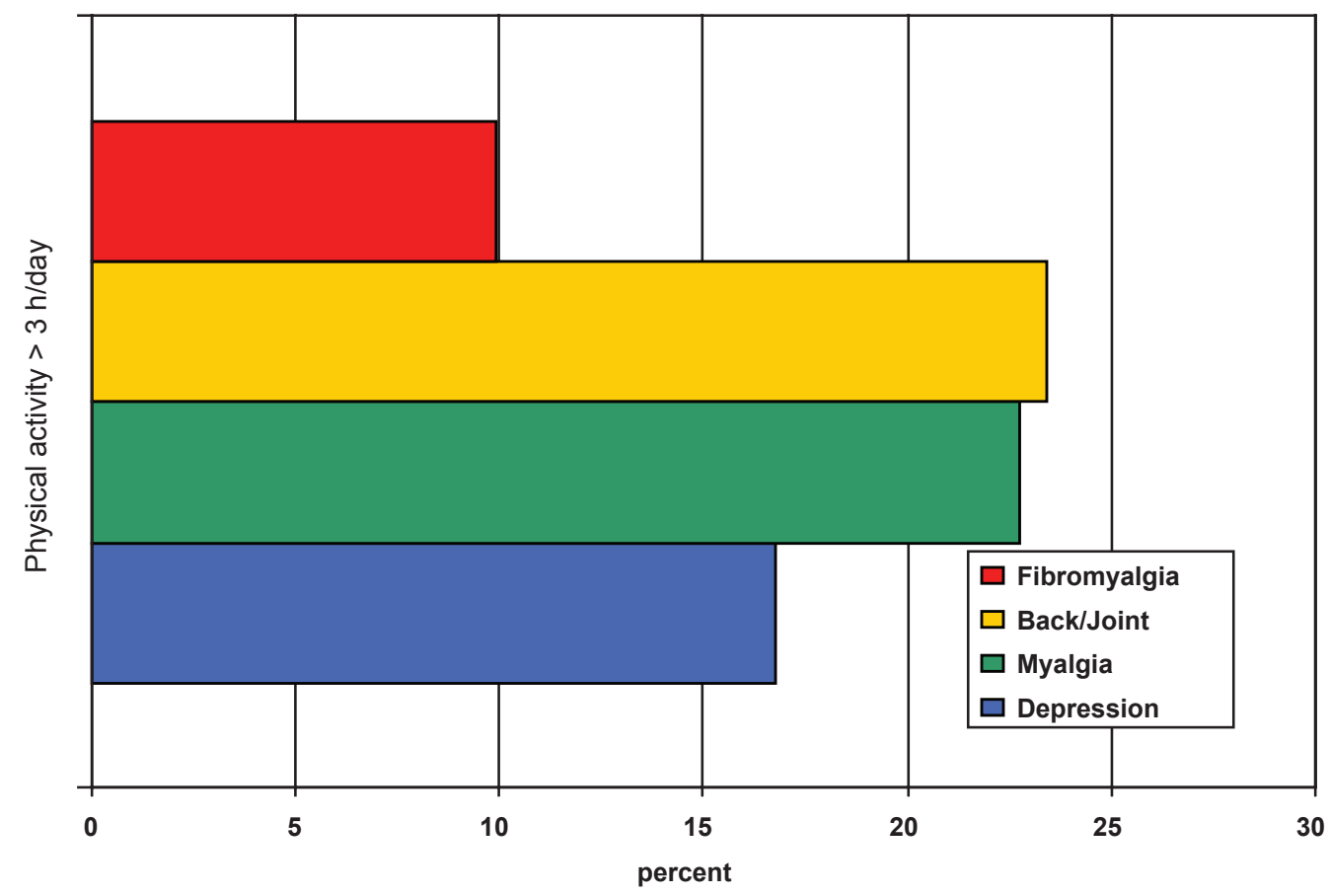

Figure 7 Present levels of physical activity in patients with fibromyalgia and patients with other somatic disorders.

$85 \%$ of patients with depression answering the item "do you have ongoing pain (yes/no). Similar results were obtained using the CPRS item "aches and pain": a score $>1$ of a maximum of 3 points (where $0=$ no pain, $1=$ mild pain, $2=$ moderate pain, and $3=$ severe pain) was reported by $100 \%$ of patients with FM, $98 \%$ of patients with myalgia, $98 \%$ of patients with back/joint diagnoses, and $87 \%$ of patients with depression (Table 2).

\section{Pain intensity}

Pain, measured in mm VAS, was noted in all patients, varying from 30-85 mm VAS. The intensity of present pain, worst and least pain during the preceding two months was highest in the FM patients, followed by patients with spinal or joint disorder, patients with myalgia, and patients with depression (Table 2).

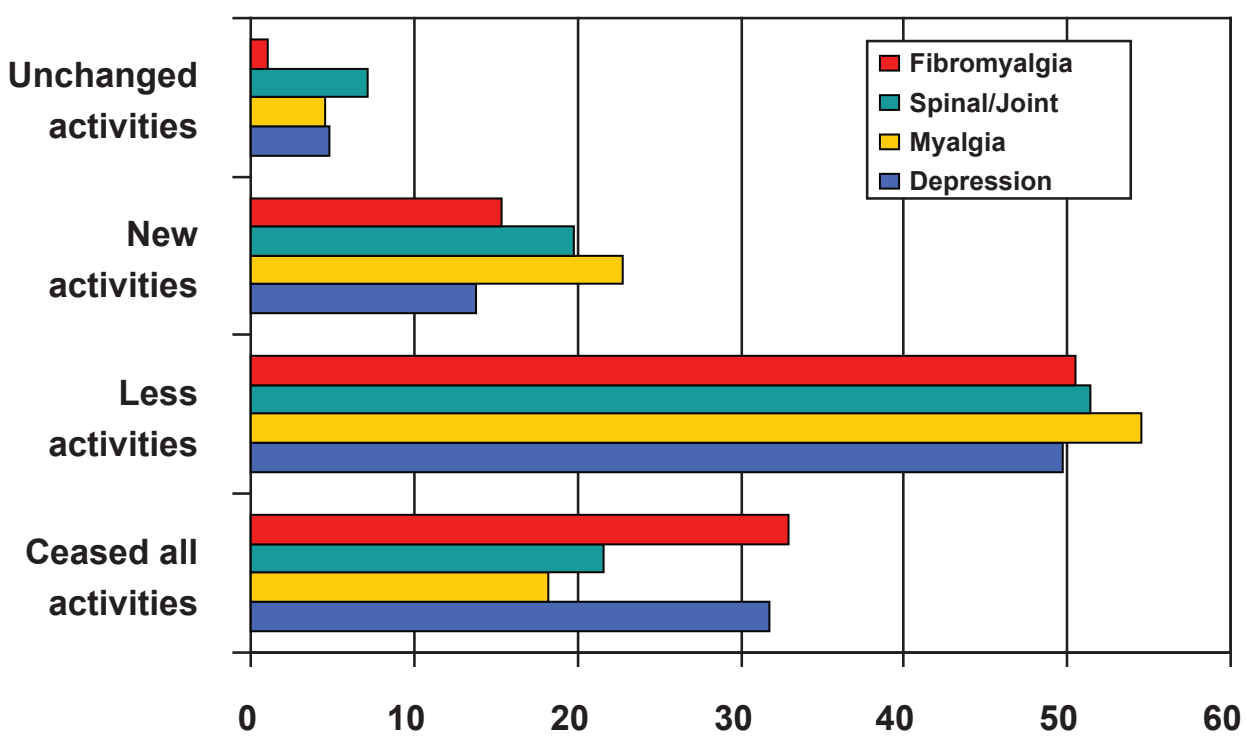

Figure 8 Changes of leisure activities in the four patient groups. 
Table 2 Pain variables: Percent of patients with present pain;VAS least, worst, now $(\mathrm{mm})$, and percent of patients with local debut of pain

\begin{tabular}{llllll}
\hline Pain variables & Fibromyalgia & Myalgia & Back/Joint diagnoses & Depression & Significance $\mathbf{p}$ (chi-square test) \\
\hline Present pain (\%) & 100 & 100 & 98.2 & 85.0 & $\mathrm{P}<0.000 \mathrm{I}$ \\
VAS least (mm) & 45.5 & 33.9 & 34.4 & 29.7 & $\mathrm{P}<0.000$ I \\
VAS worst (mm) & 86.8 & 78.8 & 79.8 & 73.7 & $\mathrm{P}<0.000$ I \\
VAS now (mm) & 63.7 & 50.2 & 52.6 & 44.1 & $\mathrm{P}<0.000$ I \\
Local pain debut (\%) & 85.9 & 97.7 & 100 & 52.0 & $\mathrm{P}<0.000$ I \\
\hline
\end{tabular}

Notes: Patient subgroups based on diagnoses of fibromyalgia, myalgia, diseases in spine/joints, and depression. Abbreviation: VAS, visual analogue scales.

\section{Pain-free periods}

Ceaseless pain was reported by $73 \%$ of the FM patients, $54 \%$ of patients with back/joint diagnoses, $43 \%$ of the patients with myalgia, and $35 \%$ of patients with depression (Figure 9).

Pain-free days were reported by $7 \%$ of patients with FM, $23 \%$ of patients with back/joint diagnoses, $25 \%$ of patients with myalgia, and $30 \%$ of patients with depression.

In summary, patients with FM were significantly more troubled with pain and had fewer pain-free intervals than patients with other pain conditions.

\section{What is the pattern of pain distribution in FM patients compared with patients diagnosed with myalgia, back/joint disorders, or depression?}

The distribution of pain in patients with depression at a frequency above $50 \%$ was only lower dorsal neck, upper shoulders and lumbosacral back (Figure 10). These patients had no pain in the anterior portion of the body.

The corresponding distribution among patients with spinal or joint diagnoses was: lower posterior neck, shoulders except lower left shoulder, upper thoracic spine, lumbosacral spine and right hip region.

Patients with myalgia marked pain $(>50 \%)$ in the same regions as those with spinal or joint diagnoses. Exceptions were the upper thoracic spine and the anterior and posterior aspects of the right upper arm.

In patients with FM, pain was distributed to all regions of the anterior and posterior body except the anterior abdomen (belly), anterior and posterior upper legs (thighs), and posterior lower legs.

\section{How is FM patients' health-related quality of life?}

All patient groups had significantly lower T-scores for health-related quality of life compared with age- and

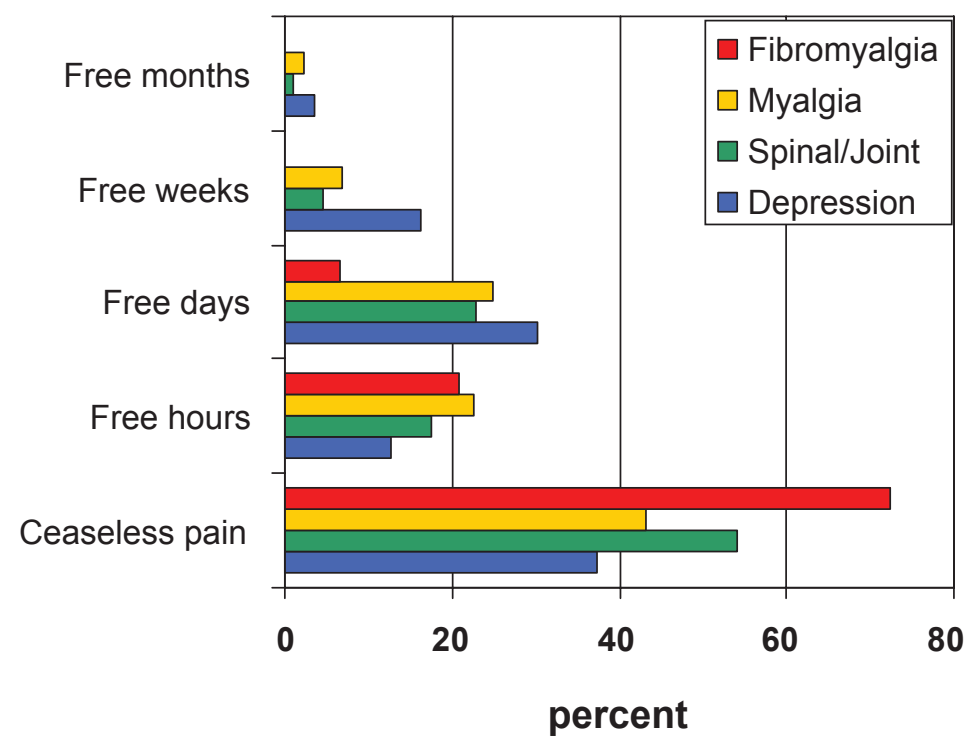

Figure 9 Pain-free periods in the four patient groups. 


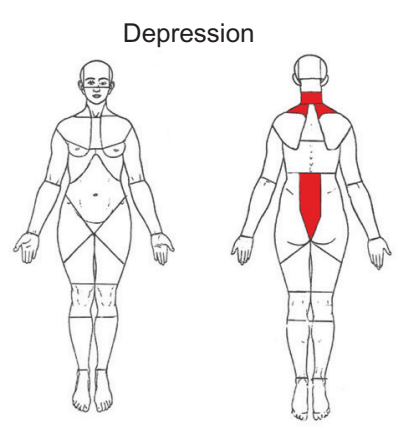

Spinal or joint diagonses
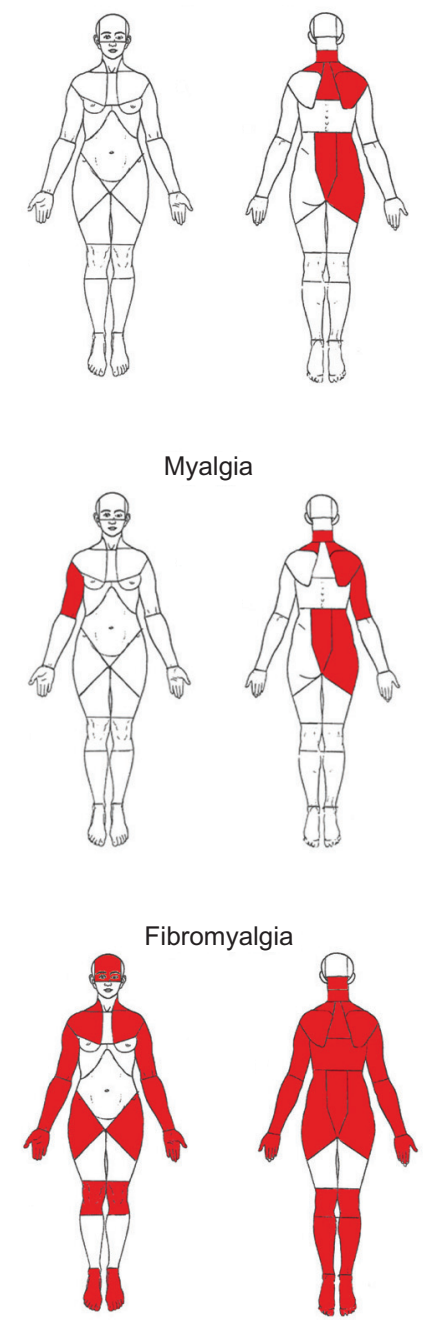

Figure 10 Distribution of pain areas reported by more than $50 \%$ in patients with fibromyalgia (FM), myalgia, back/joint diagnoses, and depression.

gender-related Swedish norms in all eight dimensions of the SF36. Among these groups, patients with FM had lower scores than the three other patient groups for physical functioning, physical role, bodily pain, and general health. Patients with depression had lower scores than the remaining patient groups for social function, emotional role, and mental health (Figure 11).

\section{What proportion of the FM patients had a local pain condition before FM developed?}

Patient records included information on each patient's pain history, such as local debut, and debut after accident or surgery. Retrospectively from this data, local debut of pain was noted for $85 \%$ of the FM patients, compared with $52 \%$ for patients with depression, $98 \%$ for those with myalgia, and $100 \%$ in patients with spinal/joint disorders (Table 2).

\section{Discussion}

Wolfe $^{32}$ has described and illustrated the widespread and continuous distribution of pain in patients with FM, using pain drawings of several individual patients. The drawings fit well with this study's superimposed illustrations (Figure 10). The distribution of pain in FM accords with Wolfe's initial case-wise descriptions. The present study emphasizes merely a "basic pattern", using only the areas with pain marked by more than half the patients. In this way, the more "unusual" marked pain areas are "filtered" away, enabling a main pattern to emerge.

To summarize the findings on symptoms, FM patients had higher scores for aches and pains, fatigue, diminished sleep, perceived muscular tension, while the depression group had higher scores for the other symptoms (Figure 2); the FM patients came between the depression patients and myalgia/back/joint patients.

Three items were common in both patients with depression and those with FM: lassitude (lack of initiative), concentration difficulties, and reduced appetite. These same symptoms are also criteria for the diagnosis of "depressive episode" in DSM-IV. If these symptoms regularly occur in FM, they may lead the physician towards a possibly erroneous diagnosis of depression.

Our results indicate that patients with FM, compared with patients with depression, have characteristic symptom profiles with dominance of pain, fatigue, disrupted sleep, and muscular tension. For patients with depression the dominant symptoms were fatigue, inner tension, lack of initiative, muscular tension, and concentration difficulties. For patients with myalgia and/or back/joint diagnoses, the symptom pattern was similar to that for FM patients, ie, with similar, but less intense symptom dominance as in FM. The occurrence of overlapping symptoms in FM and depression may mislead the physician towards psychiatric diagnoses in FM patients.

When considering the symptoms the other way round and studying the DSM-IV criteria for depression, we noted that 


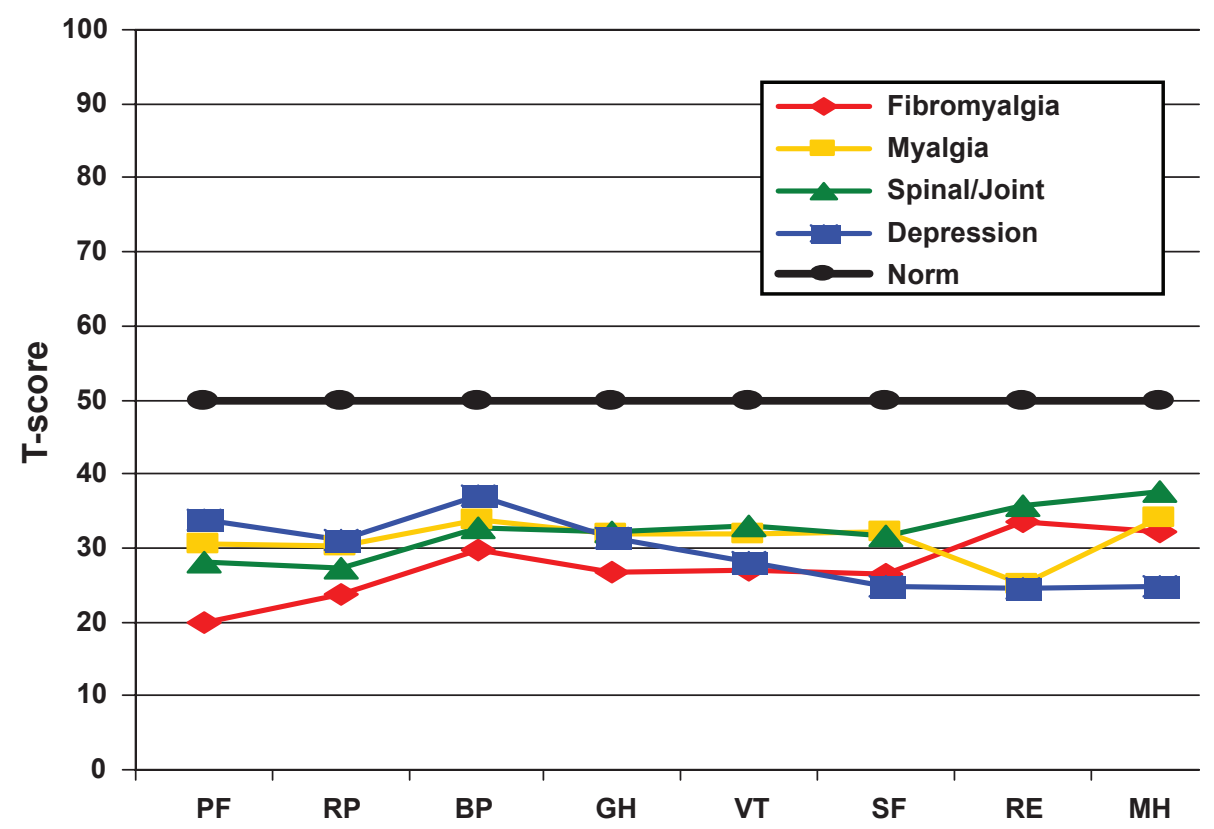

Figure II Reported health-related quality of life in patients with fibromyalgia, myalgia, back/joint diagnoses, and depression.

Notes: Eight dimensions: physical functioning (PF), role limitations due to physical problems (RP), bodily pain (BP), general health (GH), vitality (VT), social functioning (SF), role limitations due to emotional problems $(\mathrm{RE})$, mental health $(\mathrm{MH})$. The values are compared with corresponding Swedish norms controlled for age and gender. The norm has a T-score $=50$.

patients with FM met three criteria for depression: reduced energy, reduced sleep, and concentration difficulties, to the same extent as patients with depression; but not the other six criteria: pessimistic thoughts, loss of interest, sadness, inner tension, changed appetite, and suicidal thoughts. This result indicates overlapping, but not identical criteria for depression in patients with FM, and may increase the risk that patients with FM are over-diagnosed as having depression.

Our results indicate that self-reported diagnostic criteria for major depression tend to lead to overdiagnosis with depression in patients with FM compared with clinical diagnoses. Almost all patients with FM reported reduced energy, reduced sleep, and concentration difficulties, ie, three of nine criteria for the diagnosis of major depression according to DSM-IV. For this diagnosis five criteria are needed. One limitation, however, is the comorbidity of depression within all "somatic" groups.

A comment on the findings regarding criteria for DSM-IV personality disorders is that, as expected, patients with depression met more of the criteria for all DSM-IV personality disorders and that FM patients did not differ in this regard from those with other somatic disorders. This finding indicates that there is no psychiatric vulnerability in FM expressed as personality disorders. Gerdle and Elert ${ }^{33}$ explain that no clear indication for psychopathology has been demonstrated for FM, but that a link to depression or depressive symptoms has been found in many studies, as well as higher levels of psychological stress. Patients in this study with somatic diagnoses more often had personality traits of "maintaining order", compared with patients with depression who more often had "borderline" personality traits. These 'borderline' patients can be understood as having a weaker and less defined personal identity than patients with somatic diagnoses; but note that these personality traits are clearly below the cutoff levels of any personality disorder in DSM-IV.

All four patient groups had significantly lower qualityof-life scores in all eight domains of the SF36, compared with the Swedish norm, and when controlled for age and gender.

Among the four groups, patients with FM had lower T scores in the domains of physical functioning, physical role function, bodily pain, and general health than the three other groups, including patients with depression. On the other hand, the patients with depression had lower T-scores than all three "somatic" groups in the "mental" domains: social functioning, emotional role functioning, and mental health. These findings are logical, including the finding that FM patients experienced the lowest quality of life in the "somatic"domain, which is not unexpected, because it has been shown in earlier studies ${ }^{33}$ and is supported by clinical empiricism. 
Limitation of common activities in FM patients has been described ${ }^{33,34}$ and the present findings are in line with this finding. But this study also shows that our FM patients experienced more disability than our other patients with longstanding somatic pain conditions, and those with depression (Figure 5).

It is natural that all patients with myalgia or back/joint diagnoses report that the pain started locally. However finding that $85 \%$ of the FM patients also report that pain started locally, is interesting because it influences the view of the pathophysiology of FM. There are, however, earlier reports of FM developing from local pain, eg, following cervical spine injuries. ${ }^{35}$

A common opinion of FM patients with long-lasting pain problems and inability to work is that they were already avoiding physical activity at school or tended to avoid exercise as an early habit. This opinion has no support in the present results; since $70 \%-85 \%$ of our subjects reported that before the age of 30 they were engaged in active training or exercised at competition level (Figure 6). Moreover, only about 10\% indicated that they were not physically active before 30 years of age. Thus measures taken to increase physical activity among young people will not, despite their other advantages, solve the pain problems of older patients.

The finding that only one of ten FM patients reported current physical activity of more than three hours daily fits with earlier studies reporting intolerance of muscular exertion in $\mathrm{FM}^{7,33}$ (Figure 7). More patients with myalgia or back/joint diagnoses were physically active for three hours or more a day.

There are several difficulties for a FM patient with insufficient CNS attenuation of pain-signal inputs, perhaps aggravated in a particular hormonal internal milieu due to female gender and/or stress, so that afferent signals from the musculoskeletal system, not normally painful are perceived as painful, eg, when muscles and tendons are loaded at levels normally not triggering a prolonged pain response.

Because pharmacological treatment has limited success, ${ }^{13}$ effective rehabilitation methods have been sought. Rehabilitation programs have been developed and published, ${ }^{36-40}$ some showing effects on functioning. A rationale stemming from the present results (Figure 9) would be a possible rehabilitation goal of not using the category "ceaseless pain", but rather pain-free hours and, as a next step, pain-free days.

Theoretically, such a goal can often be achieved with rehabilitation programs including training to avoid elicitation of prolonged pain responses, eg, by identifying and eliminating situations when pain responses are regularly evoked ${ }^{41}$ by setting limits, ${ }^{42}$ by developing body awareness, ${ }^{42}$ by training in "using pain as a guide" to stop a loading activity before a full pain response is triggered ${ }^{42}$ training in using work movements with less muscular activity ("muscular tension") and thereby less load on muscles and tendons, ${ }^{43}$ by using various relaxation techniques such as EMG feedback, preferring low-resistance exercises to high-resistance, and preferring cardiovascular endurance training methods to high-velocity training.

\section{Summary of major results and conclusions}

1. More than $95 \%$ of patients with FM had symptoms of aches and pains, tiredness, and muscular tension.

2. Reduced sleep was more evident in patients with FM than in patients with other somatic disorders and patients with depression.

3. Almost all patients with FM reported reduced energy, reduced sleep, and concentration difficulties, three of five criteria required for the diagnosis of major depression according to DSM-IV. Thus FM patients may "need" only two more criteria to meet the diagnostic criteria for a diagnosis of major depression according to DSM-IV, meaning an overlap between symptoms in FM and in depression, which may lead the physician to a "premature" diagnosis of depression rather than FM.

4. Patients with FM did not meet more criteria for personality disorder than patients with the other somatic pain conditions studied. The most common dimension of "personality traits" of somatic pain conditions seen as a group was the "obsessive compulsive", but at a level clearly below that indicating a personality disorder.

5. More patients with FM experienced disabilities than the other patient groups studied, the most common being in the mobility and domestic-life categories.

6. The present patient groups did not have low physical activity before the age of 30 years and there was a difference between the groups studied.

7. FM patients were currently less physically active than the other patients with somatic pain.

8. Almost no FM patients had unchanged leisure activities and more FM patients had ceased all their earlier leisure activities than the other somatic-pain patients studied.

9. FM patients perceived higher pain intensities than patients with the other somatic pain conditions studied and the depression patients. Three-quarters of the FM patients felt ceaseless pain, and none had pain-free months or weeks. Only about one of 15 FM patients had pain-free days. 
10. The distributions of self-estimated pain in FM patients, recorded as pain-marked areas by over half the patients, differ from those of patients with myalgia, spinal/joint pain and depression. In FM the distribution of pain $(>50 \%)$ in the patients studied covers the whole body except the thighs, anterior part of the lower legs and lower thorax, and abdomen. The depression patients studied marked pain $(>50 \%)$ on only the dorsal aspect of the body: lower neck, upper shoulders and lumbosacral back regions. In the patients $(>50 \%)$ with spinal/joint pain the distribution also covered the upper-thoracic spine, and the right side lower-shoulder and hip region. Our myalgia patients $(>50 \%)$ marked pain in the above regions, except the upper thoracic spine but also the anterior and posterior right upper arm.

11. All patients had lower health-related quality of life in all dimensions studied than did age- and gender-matched controls. FM patients had the lowest scores in the dimensions of physical functioning, role due to physical factors, bodily pain and perceived general health; while the depression patients had the lowest scores for social functioning, role due to emotional factors and perceived mental health.

12. According to patient medical records, $85 \%$ of the FM patients reported that their pain condition started with local pain.

\section{Acknowledgement}

The study was supported by the Karolinska Institutet, Stockholm. The authors report no conflicts of interest in this work.

\section{References}

1. Linder J, Schüldt Ekholm K, Brodda Jansen G, Lundh G, Ekholm J. Long-term sickleavers with difficulty in resuming work: comparisons between psychiatric-somatic comorbidity and monodiagnosis. Int $J$ Rehabil Res. in press 2008.

2. Liedberg G, Henriksson C. Factors of importance for work disability in women with fibromyalgia. Arthritis Care Res. 2002;47:266-274.

3. Henriksson CM, Liedberg GM, Gerdle B. Women with fibromyalgia: work and rehabilitation. Disabil Rehabil. 2005;27:685-694.

4. Bennett RM, Jones J, Turk DC, Russell IJ, Matallana L. An internet survey of 2,596 people with fibromyalgia. BMC Musculoskelet Disord. 2007;8:27.

5. Staud R, Cannon RC, Mauderli AP, Robinson ME, Price DD, Vierck CJ Jr. Temporal summation of pain from mechanical stimulation of muscle tissue in normal controls and subjects with fibromyalgia syndrome. Pain. 2003;102:87-95.

6. Sörensen J, Graven-Nielsen T, Henriksson KG, Bengtsson M, Arendt-Nielsen L. Hyperexcitability in fibromyalgia. J Rheumatol. 1998;25:152-155.

7. Kosek E, Ekholm J, Hansson P. Modulation of pressure pain thresholds during and following isometric contraction in patients with fibromyalgia and in healthy controls. Pain. 1996;64:415-423.

8. Arendt-Nielsen L, Graven-Nielsen T. Muscle pain: sensory implications and interaction with motor control. Clin J Pain. 2008;24:291-298.
9. Henriksson KG. Fibromyalgia--from syndrome to disease. Overview of pathogenetic mechanisms. J Rehabil Med. 2003;(41 Suppl):89-94.

10. Henriksson KG, Mense S. Pain and nociception in fibromyalgia: clinical and neurobiological considerations on aetiology and pathogenesis. Pain Rev. 1994;1:245-260.

11. Bennett RM. Adult growth hormone deficiency in patients with fibromyalgia. Curr Rheumatol Rep. 2002;4:306-312.

12. Anderberg UM, Liu Z, Berglund L, Nyberg F. Plasma levels on nociceptin in female fibromyalgia syndrome patients. $Z$ Rheumatol. 1998;57(Suppl 2):77.

13. Okifuji A, Turk DC. Fibromyalgia syndrome: prevalent and perplexing. Pain Clin updates. 2003;XI:1-4.

14. Giamberardino MA. Update on fibromyalgia syndrome. Pain Clin Updates. 2008;XVI:1-6.

15. Fishbain DA, Cutler R, Rosomoff RS. Chronic pain-associated depression: antecedent or consequence of chronic pain? A review. Clin J Pain. 1997;13:241-248.

16. Linder J, Svensson O. The impact of pain and depression on assessment of rehabilitation need: a cross-sectional study in long-term sick-listed patients. Int J Rehabil Res. 2007;30:255-260.

17. Crofford LJ, Mease PJ, Simpson SL, et al. Fibromyalgia relapse evaluation and efficacy for durability of meaningful relief(FREEDOM): a 6-month, double-blind, placebo-controlled trial with pregabalin. Pain. 2008:136;419-431.

18. Arnold LM, Lu Y, Crofford LJ, et al. A double-blind multicenter trial comparing duloxetine with placebo in the treatment of firbromyalgia patients with or without major depressive disorder. Arthritis Rheum. 2004:50;2974-2984.

19. Bengtsson A, Henriksson KG, Jorfeldt L, Kagedal B, Lennmarken C, Lindström F. Primary fibromylgia. A clinical study of 55 patients. Scand J Rheumatol. 1986;15:340-347.

20. Wolfe F, Smythe HA, Yunus MB, et al. The American College of Rheumatology 1990 critera for the classification of fibromyalgia. Arthritis Rheum. 1990;33:160-172.

21. McCain GA. The clincal features of the fibromyalgia syndrome. In: Vaeroy H, Mersky H, editors. Progress in fibromyalgia and myafascial pain. Pain Research and Clinical Management. Amsterdam: Elsevier;1993;6:195-215.

22. Cöster L, Kendall S, Gerdle B, Henriksson C, Henriksson KG, Bengtsson A. Chronic widespread musculoskeletal pain - a comparison of those who meet criteria for fibromyalgia and those who do not. Eur J Pain. 2008;12:600-610.

23. Svanborg P, Åsberg M. A new self-rating scale for depression and anxiety states based on the Comprehensive Psychopathological Rating Scale. Acta Psychiatr Scand. 1994;89:21-28.

24. American Psychiatric Association. Diagnostic and statistical manual of mental disorders. 4th Ed. Washington, DC: American Psychiatric Association; 1994.

25. Svanborg P, Ekselius L. Self-assessment of DSM-IV criteria for major depression in psychiatric out and inpatients. Nord J Psychiatry. 2003;57:291-296.

26. Sullivan M, Karlsson J, Ware JE. [SF-36. Hälsoenkät, manual och tolkningsguide.] In Swedish. SF-36, Health survey, manual and interpretation guide. Summary in English. Gothenburg: Health Care Research Unit, Gothenburg University; 1994.

27. Sullivan M, Karlsson J. The Swedish SF-36 Health survey III. Evaluation of criterion-based validity: results from normative population. J Clin Epidemiol. 1998;51:1105-1113.

28. Landstad B, Schüldt K, Ekholm J, Broman L, Bergroth A. Woman at work despite ill-health: diagnoses and pain before and after personnel support. A prospective study of hospital cleaners/home help personnel with comparison groups. J Rehabil Med. 2001;33:216-224.

29. 4D Inc., 4D 2004, versoin 8.0.2. San Jose, CA: 4D Inc., 2005. Available from: httpi//www.4d.com/world.

30. JMP version 7 for Windows (2007). Cary, North Carolina: SAS Institute Inc.

31. World Health Organization. International classification of functioning, disability and health - ICF. Geneva: WHO; 2001. 
32. Wolfe F. Fibromyalgia and problems in classification of musculoskeletal disorders. In: Vaeroy H, Mersky H, editors. Progress in fibromyalgia and myafascial pain. Pain Research and Clinical Management, Vol 6 , Amsterdam: Elsevier;1993. p. 217-235.

33. Gerdle B, Elert J. Disability and impairment in fibromyalgia syndrome: possible pathogenesis and etiology. Crit Rev Phys Rehabil Med. 1995;7:189-232

34. Henriksson C, Burckhardt C. Impact of fibromyalgia on everyday life: a study of women in the USA and Sweden. Disabil Rehabil. 1996;18:241-248.

35. Buskila D, Neuman L, Vaisberg G Alkalay D, Wolfe F. Increased rates of fibromyalgia following cervical spine injury. A controlled study of 161 cases of traumatic injury. Arthritis Rheumatol. 1997;40:446-452.

36. Goldenberg DL, Buckhardt C, Crofford L. Management of fibromylgia syndrome. JAMA. 2004;292:2388-2395.

37. Lemstra M, Olszynski WP. The effectiveness of multidisciplinary rehabilitation in the treatment of fibromyalgia: a randomized controlled trial. Clin J Pain. 2005;21:1666-1674.
38. Sim J, Adams N. Systematic review of randomized controlled trials of nonpharmacological interventions for fibromyalgia. Clin J Pain. 2002;18:324-336.

39. Karjalainen K, Malmivaara A, van Tulder M, et al. Multidisciplinary rehabilitation for fibromyalgia and musculoskeletal pain in working age adults. Cochrane Database Syst Rev. 2000;2:CD001984.

40. Gustafsson M, Ekholm J, Broman L. Effect of a multiprofessional rehabilitation programme for patients with fibromyalgia syndrome J Rehabil Med. 2002;34:119-127.

41. Gustafsson M, Ekholm J, Öhman A. From shame to respect: musculo-skeletal pain patient's experience of a rehabilitation programme, a qualitative study. J Rehabil Med. 2004;36:97-103.

42. Löfgren M, Ekholm J, Öhman A. "A constant struggle" - successful strategies of women in work despite fibromyalgia. Disabil Rehabil. 2006;28:447-455.

43. Löfgren M, Broman L, Ekholm J. Does rehabilitation decrease shoulder muscle activity in fibromyalgia in work or housework tasks? An electromyographical study. Work. 2008;31:195-208. 
